\title{
Plasma Fibrinogen increase due to polymorphism rs2066865 in FGG gene as a risk factor for thrombosis in microvascular surgery
}

Drizlionoka- Gorovenko K, *1,2 Stepanovs J., ${ }^{1,2}$ Ozolina A., ${ }^{2}$ N̦ikitina-Zaķe L., ${ }^{3}$ Mamaja B. ${ }^{1,2}$

${ }^{1}$ Riga East Clinical University Hospital, Latvia

2 Riga Stradins University, Latvia

${ }^{3}$ Latvian Biomedical Research and Study Center, Latvia

Introduction: To cover tissue defects caused by trauma, burns, chronic inflammation, malignancies microvascular free flap surgery is used. Thrombosis with consequent loss of free flap is seen as detrimental sequel resulting in convalescence delay. We aimed to evaluate plasma fibrinogen increase due to single nucleotide polymorphism ( $S N P$ ) rs2066865 in Fibrinogen gamma chain ( $F G G$ ) gene, as a risk factor for thrombosis in free flap surgery.

Material and Methods: We enrolled 95 patients undergone free flap microvascular surgery in the Latvian Microsurgery Centre in observational case control study, the period 2016-2018. Single nucleotide polymorphism rs2066865 (G>A) in FGG gene, plasma fibrinogen concentration, platelet count, haemoglobin, haematocrit, white blood count (WBC), C-reactive protein (CRP) were analysed. Thromboelastometry (ROTEM) was performed for MCF (Maximal Clot Firmness) Fibtem, MCF Intem and FPR (fibrinogen/ platelet ratio) evaluation. Patients demographic data as well as history of comorbidities, family history were collected. Free flap thrombosis was defined as a primary outcome.

Results: In 19/95 (19\%) patients free flap thrombosis occurred with complete failure in 15/95 (16\%). We found 35 patients out of 95 with FGG gene mutation, 29/35 were heterozygous (A/G) and 6/35 were homozygous (A/A) for polymorphism rs2066865. Patients with SNP in rs2066865 (G>A) in FGG gene had higher plasma fibrinogen concentration (Fig. 1.) even in time period overrun 30 days (recent trauma period). In 9/35 (25.7\%) patients with FGG gene mutation thrombosis with complete flap failure eventuated. In all 35 patients with investigated SNP thromboelastometry confirmed increased MCF Fibtem values (G/A- 25.14 (9.80 $\pm \mathrm{SD})$; A/A-39.2 (21.8 $\pm \mathrm{SD})$ $\mathrm{p}=.0036$ (Cohen's $d=0.37$ ). Haematocrit and haemoglobin levels were lower in group with homozygosity (A/A) $(\mathrm{p}=.0053 ; \mathrm{p}=.0038)$ and negative correlation trend was observed between haemoglobin level and plasma fibrinogen concentration $\left(\mathrm{r}_{\mathrm{s}}=-.56\right)$ (Fig.2.). No difference among groups was found for platelet count $(\mathrm{p}=.3343)$ (Table 2.). Although a strong correlation between CRP and plasma fibrinogen concentration $\left(\mathrm{r}_{\mathrm{s}}=.73\right)$ was noted, CRP and WBC levels did not differ between groups with and without $\operatorname{SNP}(p=.0976 ; p=.0057)$.

Conclusion: Patients with SNP rs2066865 (G>A) in FGG gene had a higher plasma fibrinogen concentration, however no clear association with increased risk for thrombosis was found. Further data and larger sample size are needed.

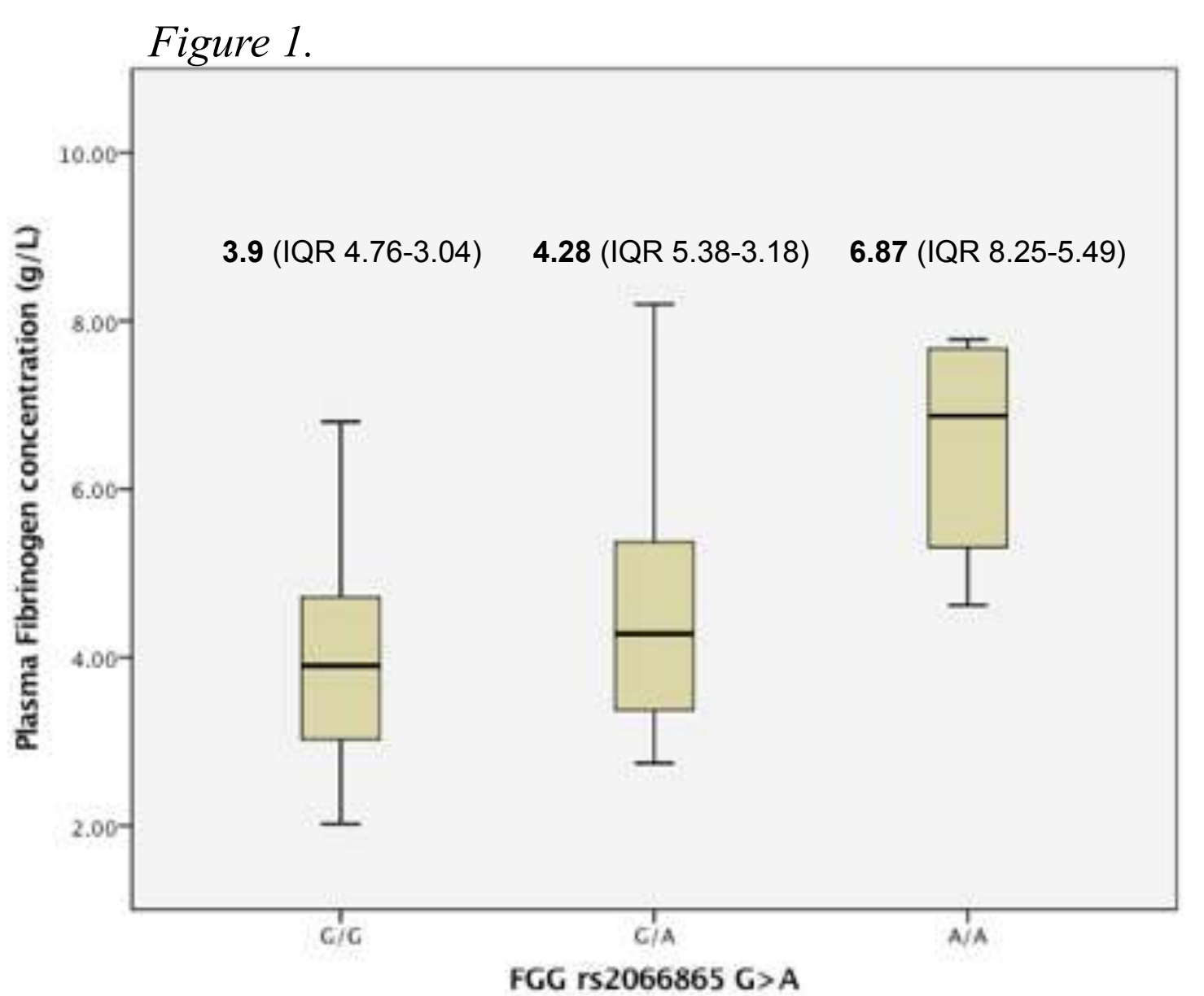

Values are median (IQR [range])

Table 1. Group characteristics (n, \%); significance level $\alpha<0.005$

\begin{tabular}{ccccc}
\hline $\begin{array}{c}S N P \\
\text { rs2066865 } \\
(\mathrm{G}>\mathrm{A}) \\
\text { in FGG gene }\end{array}$ & $\begin{array}{c}\mathrm{G} / \mathrm{G} \\
\mathrm{n}=60\end{array}$ & $\begin{array}{c}\mathrm{G} / \mathrm{A} \\
\mathrm{n}=29\end{array}$ & $\begin{array}{c}\mathrm{A} / \mathrm{A} \\
\mathrm{n}=6\end{array}$ & $p$ \\
\hline $\begin{array}{c}\text { Age, ys (M; } \\
\pm \mathrm{SD})\end{array}$ & $39.62(13.03)$ & $46.21(12.49)$ & $52.0(14.39)$ & .0105 \\
$\begin{array}{c}\text { Sex, female } \\
\text { Free flap } \\
\text { thrombosis }\end{array}$ & $7(11.6 \%)$ & $4(13.7 \%)$ & $1(16.6 \%)$ & .3626 \\
$\begin{array}{c}\text { History of } \\
\text { thrombosis }\end{array}$ & $2(3.33 \%)$ & $3(10.3 \%)$ & $2(33.3 \%)$ & .2529 \\
$\begin{array}{c}\text { Family history } \\
\text { of thrombosis } \\
\text { Smoking }\end{array}$ & $7(11.6 \%)$ & $3(10.3 \%)$ & $2(33.3 \%)$ & .4121 \\
$\begin{array}{c}\text { Recent } \\
\text { trauma, } \\
(<30 \text { days })\end{array}$ & $22(36.6 \%)$ & $8(27.5 \%)$ & $2(33.3 \%)$ & .0924 \\
\hline
\end{tabular}

Table 2. Laboratory data

\begin{tabular}{|c|c|c|c|c|}
\hline $\begin{array}{c}S N P \\
\text { rs } 2066865 \\
(\mathrm{G}>\mathrm{A}) \\
\text { in FGG gene }\end{array}$ & $\begin{array}{c}\mathrm{G} / \mathrm{G} \\
\mathrm{n}=60\end{array}$ & $\begin{array}{c}G / A \\
n=29\end{array}$ & $\begin{array}{l}A / A \\
n=6\end{array}$ & $p$ \\
\hline $\begin{array}{c}\text { Fibtem MCF, } \\
\mathrm{mm} \\
(9-25)\end{array}$ & $23.21(11.56)$ & $25.14(9.80)$ & $39.2(21.8)$ & .0036 \\
\hline $\begin{array}{c}\text { Platelet count, } \\
109 / \mathrm{L}\end{array}$ & $\begin{array}{c}319.56 \\
(126.09)\end{array}$ & $\begin{array}{c}347.14 \\
(144.81)\end{array}$ & $\begin{array}{c}341.50 \\
(108.73)\end{array}$ & .3343 \\
\hline FPR, $<42$ & $33.82(13.88)$ & $35.00(11.53)$ & $42.20(7.98)$ & .0901 \\
\hline $\begin{array}{c}\text { Intem MCF, } \\
\mathrm{mm} \\
(50-72)\end{array}$ & $66.42(7.74)$ & $69.14(6.94)$ & $66.80(15.32)$ & 4401 \\
\hline Hct, \% & $37.66(6.06)$ & 36.27 (12.73) & $30.83(6.32)$ & .0053 \\
\hline $\mathrm{Hgb}, \mathrm{g} / \mathrm{dL}$ & $12.62(2.15)$ & 13.08 (2.09) & $10.08(1.86)$ & .0038 \\
\hline WBC, 10e9/L & $7.70(2.51)$ & $8.59(3.2)$ & $11.63(5.57)$ & .0057 \\
\hline CRP, mg/L & $16.74(24.3)$ & $21.70(29.2)$ & $36.9(19.3)$ & .0976 \\
\hline
\end{tabular}

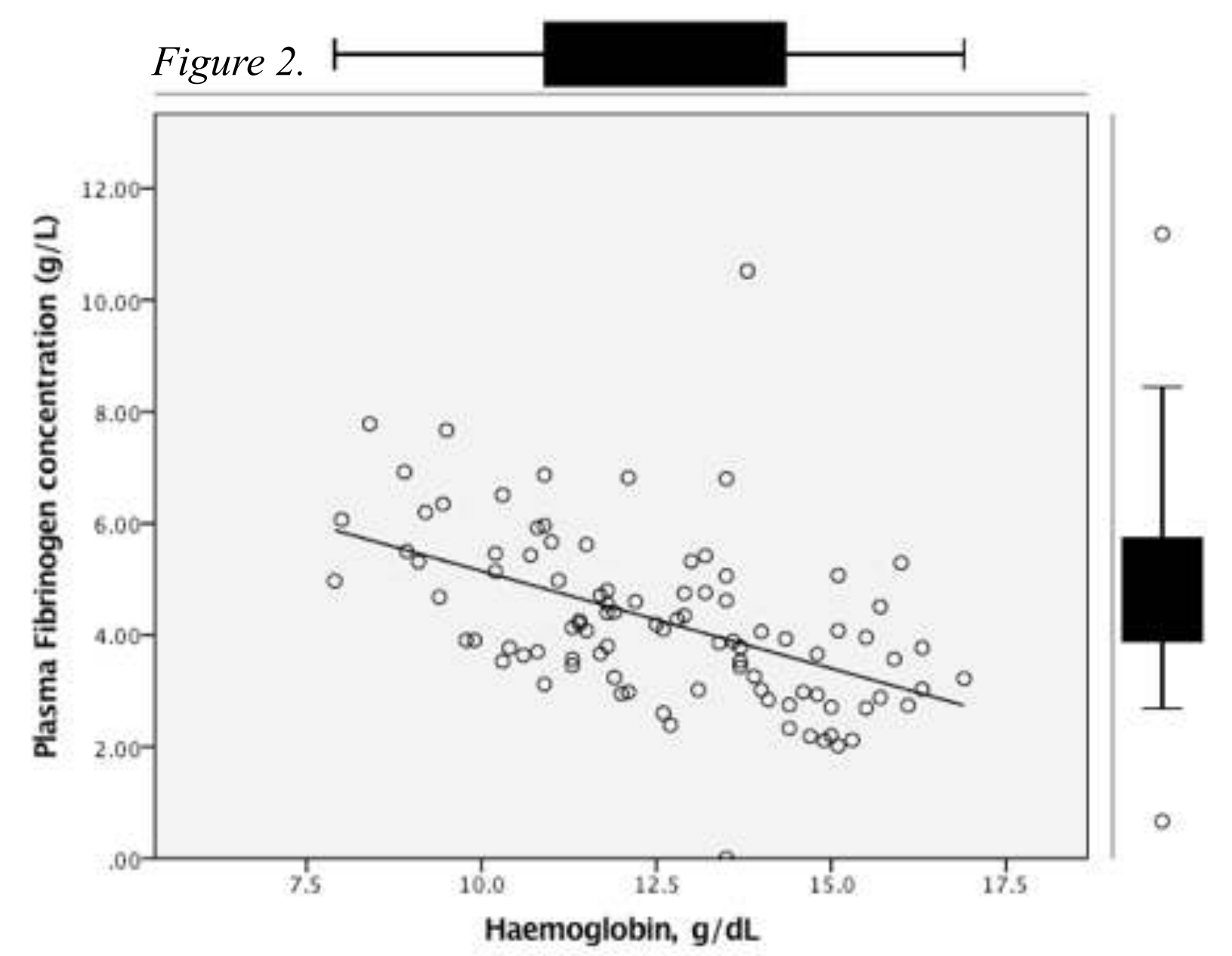

